\title{
Studies on porosity in poly( $N$-isopropylacrylamide) hydrogels for fast-responsive piezoresistive microsensors
}

\author{
Daniela Franke and Gerald Gerlach \\ Institute of Solid State Electronics, Technische Universität Dresden, 01062 Dresden, Germany \\ Correspondence: Daniela Franke (daniela.franke@tu-dresden.de) and Gerald Gerlach \\ (gerald.gerlach@tu-dresden.de)
}

Received: 22 June 2020 - Revised: 9 February 2021 - Accepted: 22 February 2021 - Published: 31 March 2021

\begin{abstract}
Porous poly $(N$-isopropylacrylamide) (PNIPAAm) hydrogels with pore diameters in the nanometer and the micrometer range were synthesized using two variations of the surfactant-based template method. We showed that smaller pore diameters lead to faster swelling and deswelling. A graphic representation of a model describing the swelling kinetics explains the assumption that the release and the uptake of water is faster for a larger specific surface area, that is smaller pore diameters. Additionally, the open-porous channel structure benefits the water transport compared to a nonporous PNIPAAm hydrogel. Sensor measurements result in response times between $36 \mathrm{~s}$ and $4 \mathrm{~min}$ also showing the importance of mechanical stability of porous hydrogels.
\end{abstract}

\section{Introduction}

Responsive hydrogels are swellable three-dimensional polymer networks. A change of the $\mathrm{pH}$ value, the concentration of analytes or the temperature of a surrounding aqueous medium leads to a volume change of these networks (Dušek and Patterson, 1968; Tanaka, 1978; Saito et al., 1993; Orakdogen and Okay, 2006) making them suitable for applications as transducers in sensors and as actuators (Guenther and Gerlach, 2009; Richter, 2009; Kuckling et al., 2003; Guenther et al., 2007; Richter et al., 2009; Richter and Paschew, 2009).

A major disadvantage of hydrogels is the slow swelling process possibly lasting for hours or even days depending on sample size and geometry. A possibility to overcome this limitation and to increase the transport rate of the aqueous medium is the incorporation of channel structures into the hydrogel. Studies on a macroporous $\mathrm{pH}$-sensitive hydrogel by Schulz et al. (2011) showed an $80 \%$ decrease of the response time of a piezoresistive microsensor compared to the nonporous hydrogel.

In this work, we synthesized $\operatorname{poly}(N-$ isopropylacrylamide) (PNIPAAm) hydrogels. Linear PNIPAAm is a well-studied polymer known for its temperature-induced change of its solubility in water. Below the so-called lower critical solution temperature $\left(\mathrm{LCST}=32^{\circ} \mathrm{C}\right)$, PNIPAAm chains are dissolved in water due to predominant polymer-solvent interactions as described in Nayak and Lyon (2005) and pictured in Scheme 1a. Above the LCST, polymer-polymer interactions overbalance the polymer-solvent interactions. The PNIPAAm chains collapse to form insoluble globules and the polymer precipitates (Nayak and Lyon, 2005; Scheme 1a). This characteristic of PNIPAAm chains translates into hydrogels as pictured in Scheme $1 \mathrm{~b}$. Below $32^{\circ} \mathrm{C}$, the predominant polymer-solvent interactions attract water molecules inside the three-dimensional crosslinked polymer network. The PNIPAAm hydrogel is in a swollen state. Above $32^{\circ} \mathrm{C}$, coiling of the PNIPAAm chains in the hydrogel occurs due to predominant polymer-polymer interactions. The volume of the hydrogel collapses, which leads to a deswollen state.

PNIPAAm hydrogels containing open-porous channel systems were prepared using two variations of the surfactantbased template method, which has been described, for example, in Hentze and Antonietti (2001), Sjöblom et al. (1996), and Pavel (2004). In variation 1 (forming PNIPAAm 1), a surfactant is dissolved in a monomer solution in a concentra- 


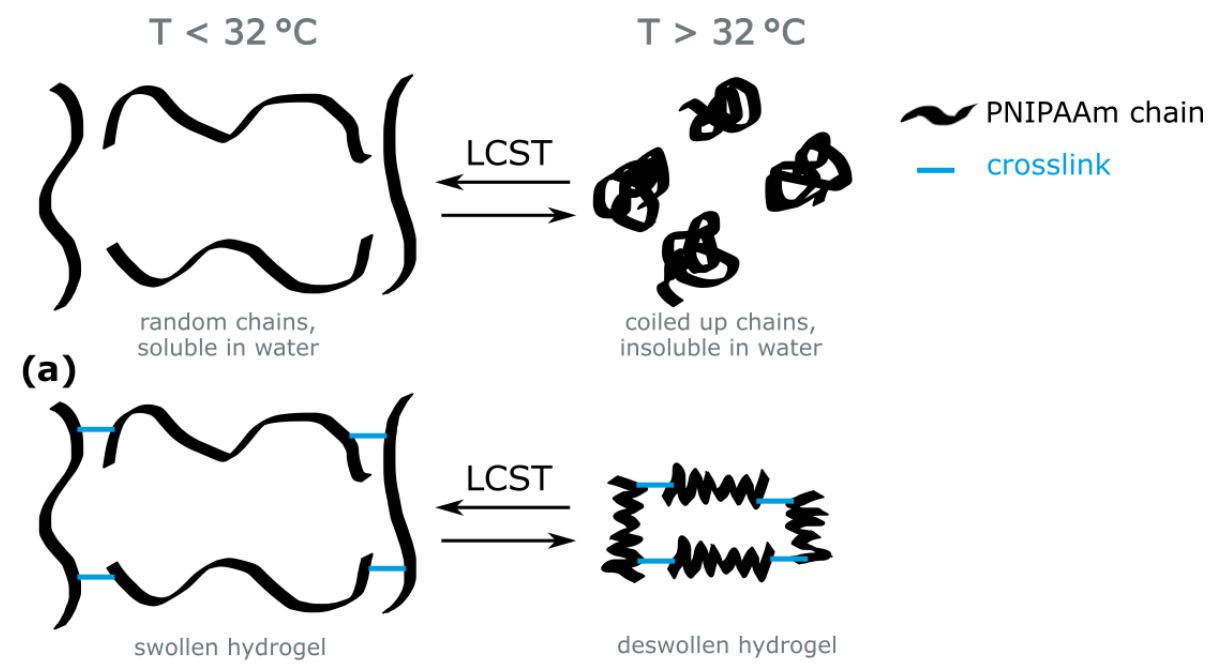

(b)

polymer-polymer interactions $\downarrow$ polymer-solvent interactions $\uparrow$ polymer-polymer interactions $\uparrow$

polymer-solvent interactions $\downarrow$

Scheme 1. Schematic representation of the behavior of (a) PNIPAAm chains and (b) PNIPAAm hydrogels in water below and above the lower critical solution temperature $\left(\operatorname{LCST}, 32^{\circ} \mathrm{C}\right)$.

tion above the critical micelle concentration forming spherical micelles with an expected diameter $<100 \mathrm{~nm}$. A nonpolar solution is added to the micelle containing template solution for variation 2 (forming PNIPAAm 2) in order to stabilize the nonpolar inside of the micelles and to form additional aggregate structures, the so-called microemulsions and lyotropic liquid crystalline phases (Tiddy, 1980).

The pores in the hydrogel polymerized in the aqueous part of the micellar solution are imprints of these micelles or liquid crystalline aggregate structures. These structures are thermodynamically stable only for a set of components at particular concentrations (Sjöblom et al., 1996). Ideally, the size and shape of the pores resemble the size and shape of the template structure. In real systems, often, a phase separation occurs during the polymerization due to the change of monomer concentration in the system (Hentze and Antonietti, 2001; Sjöblom et al., 1996; Pavel, 2004).

We already showed that using a macroporous PNIPAAm hydrogel as a transducer in a piezoresistive microsensor leads to a significant decrease of the response time for the deswelling of the hydrogel. Fast swelling on the other hand is impeded due to the restraining of the hydrogel by the flexure plate of the pressure sensor chip (Franke et al., 2017). We assume that decreasing the pore size leads to an increase of the mechanical stability of the hydrogel structure because the hydrogel content increases per volume unit. Therefore, faster swelling should also be possible in the sensor.

In this work, we present and discuss the morphology and the swelling kinetics of porous PNIPAAm 1 and PNIPAAm 2 and compare them with a conventional nonporous PNIPAAm hydrogel. We aim to show the influence of porosity and pore diameter on the rate of swelling and deswelling processes. Sensor measurements transfer the results from free swelling and deswelling experiments into an application where mechanical stability is an additional key characteristic, which will also be discussed.

\section{Methods and materials}

\subsection{Synthesis of the hydrogels}

The monomer $N$-isopropylacrylamide (NIPAAm), the crosslinker $N, N^{\prime}$-methylenebis(acrylamide) (MBA), the initiator system consisting of ammonium persulfate (APS) and $N, N, N^{\prime}, N^{\prime}$-tetramethylethylenediamine (TEMED), the surfactants Brij®L23 (polyoxyethylene (23) lauryl ether) and AOT (Aerosol ${ }^{\circledR}$ OT; bis(2-ethylhexyl) sulfosuccinate sodium salt), and the nonpolar solvent heptane were bought from Sigma-Aldrich (Munich, Germany) and were used as received for the following syntheses.

\subsubsection{Nonporous PNIPAAm}

A solution of $0.5 \mathrm{~g} \quad(4.42 \mathrm{mmol})$ NIPAAm, $0.0068 \mathrm{~g}$ $(0.044 \mathrm{mmol}) \mathrm{MBA}, 26.3 \mu \mathrm{L}$ of $0.84 \mathrm{M}$ APS solution (containing $0.02 \mathrm{mmol}$ APS), $3.33 \mu \mathrm{L}(0.02 \mathrm{mmol})$ TEMED and $3.5 \mathrm{~mL}$ deionized water was stored in sealed $1 \mathrm{~mL}$ glass tubes at $4{ }^{\circ} \mathrm{C}$ for $3 \mathrm{~d}$ to ensure the completion of the polymerization. 


\subsubsection{Porous PNIPAAm 1}

At first, $0.167 \mathrm{~g}(0.14 \mathrm{mmol})$ BrijßL23 was dissolved in $3 \mathrm{~mL}$ deionized water to form a clear micellar solution. Then, $0.5 \mathrm{~g}(4.42 \mathrm{mmol})$ NIPAAm, $0.033 \mathrm{~g}(0.21 \mathrm{mmol}) \mathrm{MBA}$ and $0.0167 \mathrm{~g}(0.07 \mathrm{mmol})$ APS were added and dissolved by stirring. After adding $7.5 \mu \mathrm{L}(0.05 \mathrm{mmol})$ TEMED, the solution was transferred to $1 \mathrm{~mL}$ glass tubes. After sealing, the tubes were stored over night at room temperature for polymerization.

\subsubsection{Porous PNIPAAm 2}

A stock solution containing $2.81 \mathrm{~g}(24.8 \mathrm{mmol})$ NIPAAm, $0.19 \mathrm{~g}(1.2 \mathrm{mmol}) \mathrm{MBA}, 0.10 \mathrm{~g}(0.44 \mathrm{mmol})$ APS and $10 \mathrm{~mL}$ deionized water was prepared at first. A total of $3 \mathrm{~g}$ thereof was mixed with heptane and AOT in the mass ratio heptane : AOT $:$ stock solution $=0.42: 0.42: 0.16$ and stirred until a clear solution was received; $63.5 \mu \mathrm{L}(0.42 \mathrm{mmol})$ TEMED was dissolved in this solution, which was then transferred to $1 \mathrm{~mL}$ glass tubes that were, after sealing, stored over night at room temperature for polymerization.

The hydrogel cylinders were removed from the glass tubes after polymerization and rinsed in deionized water for $3 \mathrm{~d}$ (nonporous hydrogel) or (in order to wash the surfactant out) $30 \mathrm{~d}$ (porous hydrogels), which was exchanged every day.

\subsection{Scanning electron microscopy (SEM)}

Air-dried fragments of the rinsed hydrogel cylinders were put onto adhesive carbon pads, contacted with conductive silver and coated with $3 \mathrm{~nm}$ platinum in the sputtering system Leica EM SCD500 (Leica Microsystems GmbH, Wetzlar, Germany). SEM images were recorded in a Neon 40EsB (Carl Zeiss Microscopy GmbH, Jena, Germany) using a Schottky field-emission electron source and an EHT (extra high tension) voltage level of the electron beam of $3 \mathrm{kV}$. Pore diameters were determined using the ImageJ software package (ImageJ, 2018).

\subsection{Swelling experiments}

For the swelling experiments, the cylindrical hydrogel samples were cut into disks of $1-2 \mathrm{~mm}$ height. The diameter was $5 \mathrm{~mm}$ in the swollen state. One hydrogel disk at a time was placed in a copper well filled with water after preheating or precooling the copper well with a Peltier element using a HAT control temperature controller from BelektroniG GmbH (Freital, Germany) and the software BelektroniG HAT Soft Pro v2.3.23. Imaging was carried out using a Bresser Microcam Pro HDMI camera (Bresser GmbH, Rhede, Germany) mounted on a microscope and the respective software Bresser MicroCamLab II. Swelling kinetics were studied by abruptly changing the temperature from 25 to $40^{\circ} \mathrm{C}$ or vice versa. For the observation of, for example, the deswelling kinetics, a hydrogel disk in a water-filled glass tube was preheated in an oven at $25^{\circ} \mathrm{C}$ for at least $24 \mathrm{~h}$. Before the measurement, the copper well was preheated to $40^{\circ} \mathrm{C}$. Then, the hydrogel disk was taken from the glass tube and put into the copper well while simultaneously the recording of the images started in time lapse ( 1 picture per $10 \mathrm{~s}$ for 2 to $4 \mathrm{~h}$ ). For the determination of the diameters of the hydrogel disks, we used the self-implemented Python-based software PIA (Python Image Analyzer). The swelling degree of the hydrogel disks was calculated as

$Q_{\mathrm{d}}=\frac{d_{t}}{d_{0}}$,

where $Q_{\mathrm{d}}$ is the swelling degree determined from the change of the disk's diameter, $d_{t}$ is the diameter of the disk at a certain time of the measurement and $d_{0}$ is the disk's diameter before the stimulus was applied. Mean values and standard deviations of the swelling degrees were calculated from triple measurements of both the swelling and the deswelling for each hydrogel sample.

Assuming isotropic swelling and deswelling, the swelling degree hypothetically determined from the sample volume $\left(Q_{\mathrm{v}}\right)$ equals the swelling degree $Q_{\mathrm{d}}$, and hence, we discuss the volume change rather than the diameter change in the following sections.

The time necessary to reach the equilibrium swelling degree (plateau of the curve) was determined graphically for each of the averaged curves.

\subsection{Sensor measurements}

The PNIPAAm 2 hydrogel was used as a transducer in a piezoresistive setup employing a pressure sensor chip. The piezoresistive sensor setup and the procedure of the sensor measurements are described elsewhere (Franke et al., 2017, Erfkamp et al., 2018).

\section{Results and discussion}

\subsection{Characterization}

Figure 1 shows the SEM images of the nonporous PNIPAAm and the porous PNIPAAm 1. As expected, the nonporous hydrogel is clearly not structured, while the porous hydrogel contains an open channel structure with pore diameters of $3.8 \pm 2.1 \mu \mathrm{m}$. The morphology of PNIPAAm 1 samples has already been presented in Franke et al. (2017). We mention it here in order to compare it with the new PNIPAAm 2.

At first sight, the morphology of the PNIPAAm 2 sample is ambiguous and, hence, we present different views of an air-dried fragment in Fig. 2. The overview of a fracture surface in Fig. 2a shows that the near-surface areas (outer layers) of the sample piece seem dense compared to the structured inner area (inner layer) of the piece. Figure $2 b$ is a close-up of the inner layer of the sample that shows larger, elliptic pores with an average ellipse axis length of $3 \mu \mathrm{m}$ and smaller 


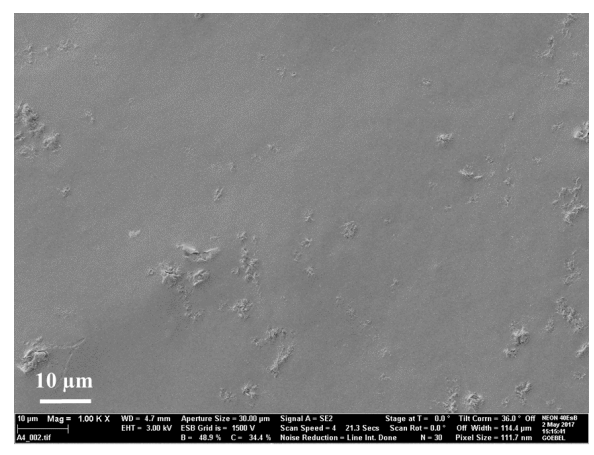

(a)

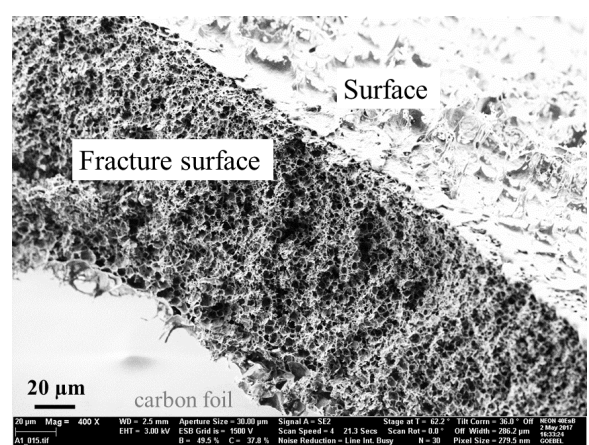

(b)

Figure 1. SEM images of (a) the nonporous PNIPAAm and (b) the porous PNIPAAm 1 sample.

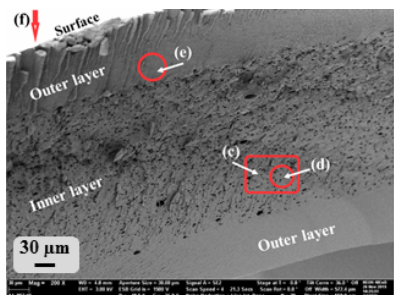

(a)

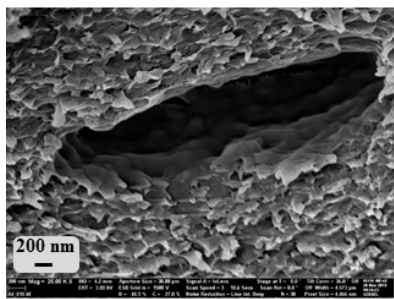

(c)

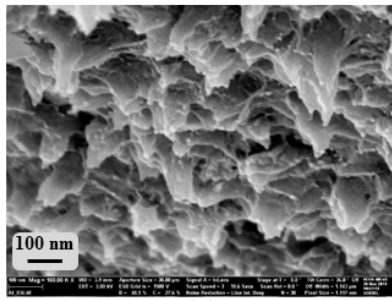

(e)

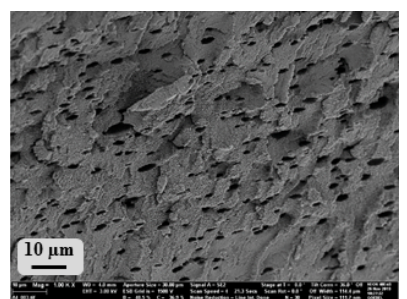

(b)

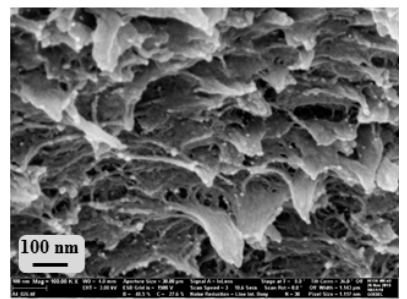

(d)

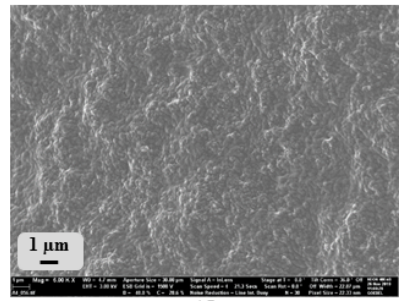

(f)
Figure 2. SEM images of several spots of porous PNIPAAm 2: overview of (a) the fracture surface and (b) the inner part of the fracture surface in (a); (c-e) close-ups; see (a); (f) surface of the hydrogel sample.

structures around these pores. A further close-up of the elliptic pores in Fig. 2c demonstrates their flaked surroundings that show openings with diameters in a range below $100 \mathrm{~nm}$ (Fig. 2d). The seemingly dense outer layer of the sample piece shows approximately the same morphology as the inner layer (Fig. 2e), while a view of the sample's surface shows a dense packaging of particles with diameters around $200 \mathrm{~nm}$ (Fig. 2f).
We found this morphology for several pieces of the same and different samples. However, different morphologies at different parts of the SEM samples were not expected because the sample pieces were cut out of disks as described above, and we expect them to be homogeneous in their inner structure. We assume that the air-drying process altered the original morphology of the wet samples. Near-surface parts collapsed more than the inner part of the samples where the original morphology is presumably conserved, while the morphology of the surface of the pieces is completely collapsed forming a dense, particle-like structure. Hence, the imaging of the morphology of PNIPAAm 2 has to be the subject of further studies. Therefore, a faster drying method, preferably freeze-drying using liquid ethane, is necessary in order to preserve the inner structure of the hydrogel sample pieces. At this point, we consider the morphology shown in Fig. $2 \mathrm{~d}$ to be the original one.

Comparison of the morphology of PNIPAAm 1 (Fig. 1b) and PNIPAAm 2 (Fig. 2d) shows that the pore diameters of PNIPAAm 1 are 1 order of magnitude larger than the pore openings in the flaked structure of PNIPAAm 2. It seems that the additional use of an organic solvent stabilized the template structure as predicted, and the phase separation of the template solution during polymerization is at least partially suppressed. The occurrence of the larger elliptical pores, however, indicates that a partial phase separation occurs because micelles of a size in the micrometer range are thermodynamically not expected in the template solution. Small pore sizes in the $100 \mathrm{~nm}$ range in the flaked part of the morphology indicate that at least parts of the template structure were preserved.

Phase separation in microemulsions and lyotropic liquid phases during synthesis is a common constraint of these surfactant-based template systems (Hentze and Antonietti, 2001; Sjöblom et al., 1996; Pavel, 2004). Further research is required regarding the possibilities of preventing phase separation of the template structure in order to preserve it in the morphology of the porous hydrogel. 


\subsection{Swelling experiments}

Figure 3 summarizes the results of the swelling experiments (deswelling at $40^{\circ} \mathrm{C}$ and swelling at $25^{\circ} \mathrm{C}$ ) and compares the swelling kinetics of nonporous PNIPAAm and porous PNIPAAm 1 and 2.

Figure 3 a shows the deswelling behavior of the nonporous and the porous PNIPAAm samples. The nonporous PNIPAAm sample shows the two-step swelling behavior that we found before in Franke and Gerlach (2020) with an initial fast decrease of the swelling degree $Q_{\mathrm{d}}$ followed by a slow further deswelling caused by the so-called skin effect. In contrast, the porous PNIPAAm samples deswell fast and in a one-step procedure. During the course of the measurements, which took up to $4 \mathrm{~h}$, the nonporous PNIPAAm never reaches the equilibrium swelling degree (plateau).

Porous PNIPAAm 1 and 2 reach the equilibrium swelling degree after 6 and $2 \mathrm{~min}$, respectively, and therefore much faster than the nonporous sample (Fig. 3c). For the swelling similar results occurred (Fig. 3b and d). While the swelling of the nonporous PNIPAAm does not reach an equilibrium during the measurement period (Fig. 3b), the porous PNIPAAm 1 and 2 reach the equilibrium swelling degree after 6 and 2 min, respectively (Fig. 3d).

Additionally, it is obvious that the volume of the nonporous hydrogel sample is reduced much more during deswelling than the volumes of the porous PNIPAAm samples (Fig. 3a) and vice versa (Fig. 3c). We explain this finding as follows. The porous PNIPAAm samples contain less deswellable/swellable hydrogel material per volume unit. Therefore, the volume of the samples is decreased/increased less. We also have evidence from our previous and ongoing research that the pore structure does not collapse during the deswelling and, therefore, does not contribute to the deswelling and swelling process. On the other hand, the entire volume of the nonporous sample is capable of deswelling/swelling. Assuming that the equilibrium swelling degree of the deswelling is reached after $54 \mathrm{~h}$ at $40^{\circ} \mathrm{C}$ (sample kept in the oven for this period), the volume of the nonporous PNIPAAm is reduced to $50 \%$ compared to its volume at $25^{\circ} \mathrm{C}$. The volumes of the porous samples change around $13 \%$ with the exception of the swelling of PNIPAAm 2 where the volume increases around $17 \%$ after decreasing only about $13 \%$ during deswelling. Incomplete conditioning of the samples might cause this difference. Another problem is the monitoring of the swelling/deswelling of a fastswelling/fast-deswelling sample that has to be put into the measurement setup from a container of different temperature. Since the swelling/deswelling takes only $2 \mathrm{~min}$ and the positioning of the samples took between 10 and $30 \mathrm{~s}$, deviations might occur during evaluation.

For a sensor application, this reduced increase and decrease of the swelling degree is an advantage regarding piezoresistive pressure sensor chips that have a limited capacity for withstanding the volume change of the transducer.

\subsection{Interpretation of the swelling kinetics}

The explanation for the fast swelling and deswelling of porous PNIPAAm 1 and 2 is presented in a schematic illustration of hydrogel volume units (presented as area units) in Fig. 4 for the release of water from the hydrogel sample (deswelling). Compared to the nonporous hydrogel in Fig. 4a, the porous hydrogel 1 in Fig. $4 \mathrm{~b}$ consists of an additional inner surface area due to the pores and, hence, more water can be released over the overall surface (outer and inner surface forming the specific surface area expressed in $\mathrm{m}^{2} \mathrm{~g}^{-1}$ ) per time unit. The channel-like structure of the openpore morphology contributes to the faster transport of water to the outside of the hydrogel volume. The reduction of the pore size is accompanied with an addition of swellable material per volume unit and leads to an increase of the inner surface area (Fig. 4c). As a result, even more water can be released per time unit. The transport of water is again supported by the channel structure of the pores.

In summary, we assume and propose that an increase of the specific surface area of a hydrogel sample is the key factor for decreasing the swelling and deswelling time. The incorporation of pores as channel structures into the hydrogel volume additionally supports the transport of water to the inside or outside of the hydrogel volume. Small dimensions of the swellable material forming the pores in the sample volume prevent the skin effect due to short paths of diffusion.

Nonetheless, we are aware of the possibility of competing mechanisms coming from fluid mechanics (especially Darcy's law). We presume that there is an optimum (minimum) of the swelling and deswelling time for a certain specific surface area/pore size. For pore sizes below that, the swelling and deswelling times may rise again due to Darcy's law. Up to now, we cannot predict that key pore size, but the surfactant-based template method to our knowledge is the only synthesis method producing small pore sizes in the nanometer range and a large variety of pore geometries required for further research.

\subsection{Sensor measurements}

Figure 5 shows the results of the sensor measurements for two sensors containing PNIPAAm 2. For both sensors the sensor signal changes with changing temperature caused by swelling (at $25^{\circ} \mathrm{C}$ ) and deswelling (at $50^{\circ} \mathrm{C}$ ) of the hydrogel. A plateau of the sensor signal is reached for each temperature step, and the level of the sensor signal stays the same after repeated swelling and deswelling processes. However, there is an obvious difference between the signal of sensor 1 and sensor 2. $\Delta V$, the difference between the output voltage at the swollen state and the output voltage at the deswollen state, is different for sensor 1 and sensor 2. Since the signal of sensor 1 is less smooth than the signal of sensor 2 , showing a pronounced overshooting at $25^{\circ} \mathrm{C}$ and recurring interference of the signal at $50^{\circ} \mathrm{C}$, we ascribe this difference 


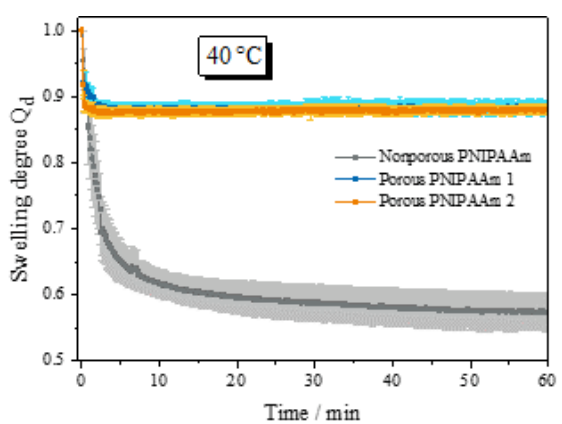

(a)

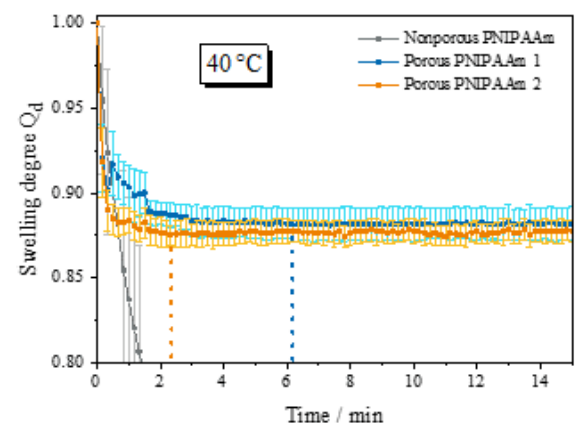

(c)

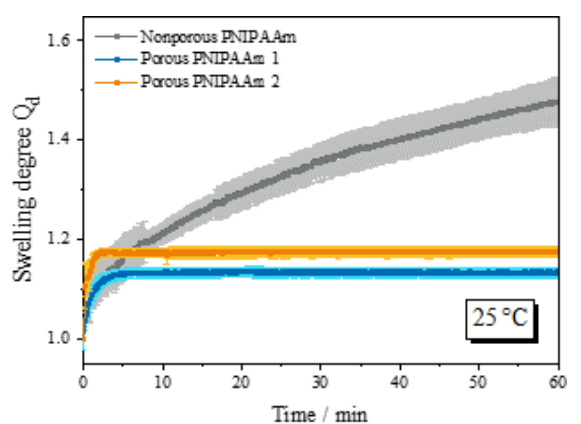

(b)

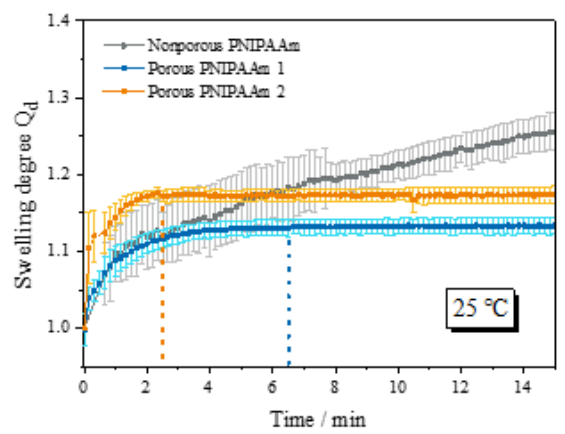

(d)

Figure 3. Results of the swelling experiments: (a, c) overview and detail of the deswelling experiments at $40^{\circ} \mathrm{C}$; (b, d) overview and detail of the swelling experiments at $25^{\circ} \mathrm{C}$; comparison of the swelling kinetics of nonporous and porous PNIPAAm samples. The dashed lines in (c) and (d) indicate the time when the swelling equilibrium (plateau) is reached.

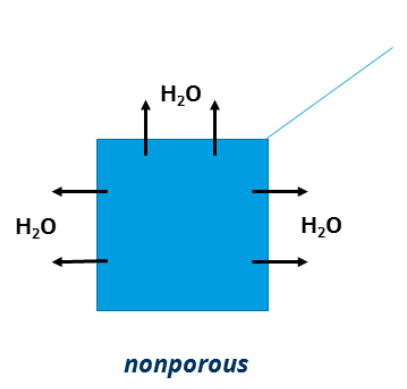

(a)

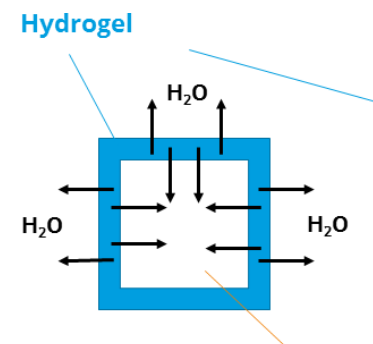

porous 1

(b)

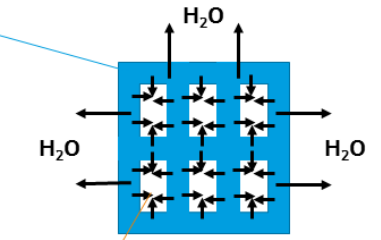

porous 2

(c)

Figure 4. Schematic illustration of the water release in nonporous and porous PNIPAAm samples.

to an inaccurate assembling of sensor 1 . The pressure sensor chip may be tilted, and hence the hydrogel cannot implement the full range of swelling on the pressure sensor chip leading to a smaller sensitivity of sensor 1 and interference during swelling and deswelling.

As shown in Table 1, the response times for the deswelling of PNIPAAm 1 and 2 at $50^{\circ} \mathrm{C}$ are similar. While PNIPAAm 1 deswells in less than $20 \mathrm{~s}$ PNIPAAm 2 is a little slower with around $40 \mathrm{~s}$. The response time for the swelling at $25^{\circ} \mathrm{C}$, however, is significantly decreased with 1.5 to 4 min when using PNIPAAm 2 compared to PNIPAAm 1, which needed
$21.5 \mathrm{~min}$. These results show that the incorporation of pores in the nanometer range into a hydrogel leads to a major improvement of the performance in a sensor application compared to macroporous and nonporous hydrogels.

\subsection{Interpretation of the sensor measurements}

Inside the pressure sensor chip, mechanical stability of the hydrogel is a key characteristic beside the capability of fast swelling and deswelling. PNIPAAm 1 lacked this mechanical stability leading to long-term swelling. As explained in 


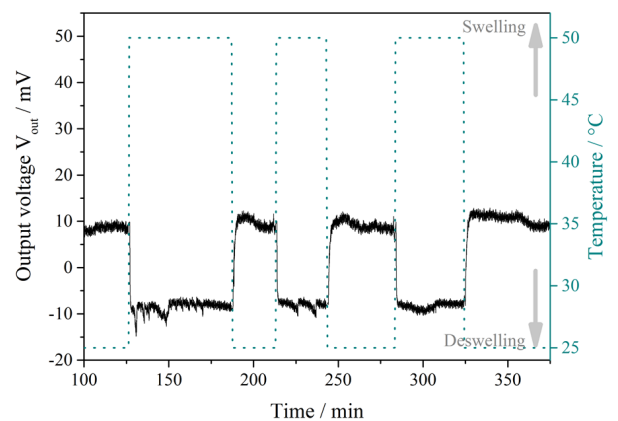

(a) sensor 1

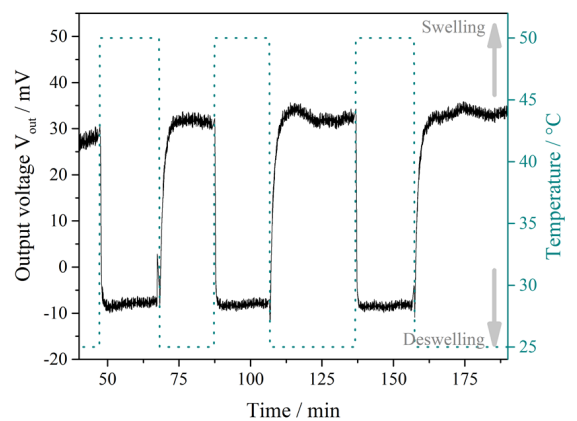

(b) sensor 2

Figure 5. Results of the sensor measurements.

Table 1. Comparison of the response times.

\begin{tabular}{lrrl}
\hline Material & Response time deswelling & Response time swelling & Reference \\
\hline nonporous PNIPAAm & $>5 \mathrm{~h}$ & $21 \mathrm{~min}$ & Franke et al., 2017 \\
PNIPAAm 1 & $<20 \mathrm{~s}$ & $21.5 \mathrm{~min}$ & Franke et al., 2017 \\
PNIPAAm 2, sensor 1 & $36 \mathrm{~s}$ & $93 \mathrm{~s}$ & this work \\
PNIPAAm 2, sensor 2 & $42 \mathrm{~s}$ & $4 \mathrm{~min}$ & this work \\
\hline
\end{tabular}

Franke et al. (2017), the porous hydrogel has to work against the flexure plate of the pressure sensor chip, which compresses the pores during deswelling. We showed that a full recovery of the porous structure is possible but takes a long time compared to the deswelling, which is supported by the flexure plate.

Figure $4 \mathrm{c}$ shows that a hydrogel with smaller pores contains additional hydrogel material per volume unit compared to the hydrogel with larger pores in Fig. 4b. This additional material serves as pillars stabilizing the porous hydrogel structure when working against the flexure plate. As a result, PNIPAAm 2 swells much faster in the sensor using both the increased mechanical stability due to the additional hydrogel material per volume unit and the increased swelling kinetics due to porosity.

The response time for the deswelling of PNIPAAm 2 is slightly increased compared to PNIPAAm 1, which can also be explained with the additional hydrogel material per volume unit. The flexure plate of the pressure sensor chip helps press the water out of the hydrogel. Due to lower mechanical stability because of larger pores, this effect is more pronounced for PNIPAAm 1 than for PNIPAAm 2. PNIPAAm 2 contains more hydrogel material per volume unit, which puts a force against the flexure plate. Therefore, the supporting role of the flexure plate during deswelling is less pronounced, and the response time for the deswelling increases compared to PNIPAAm 1.

\section{Conclusions and outlook}

The surfactant-based template method using a nonpolar solvent for stabilizing the template structure generates smaller pores in the hydrogel because phase separation is (at least partially) suppressed. Smaller pore diameters imply larger specific surface areas. Porous PNIPAAm hydrogels can therefore release or take up more water per time unit than nonporous PNIPAAm of the same volume. Our work showed that smaller pores lead to faster swelling and deswelling. The reasons for this behavior, namely a large specific surface area and the pore channel system, were described in a schematic model. Sensor measurements using the PNIPAAm 2 hydrogel showed that the decrease of the pore size adds to the mechanical stability and, therefore, leads to faster swelling behavior in the sensor compared to PNIPAAm 1, while the response time for the deswelling is still small.

Code and data availability. Data are available upon request.

Author contributions. DF conceptualized and administrated the project, investigated the research subject, visualized the data, and wrote the original draft of the manuscript. GG supervised the project and reviewed and edited the manuscript.

Competing interests. The authors declare that they have no conflict of interest. 
Special issue statement. This article is part of the special issue "Dresden Sensor Symposium DSS 2019". It is a result of the "14. Dresdner Sensor-Symposium”, Dresden, Germany, 2-4 December 2019.

Acknowledgements. The authors would like to thank Michael Göbel (Leibniz Institute of Polymer Research, Dresden, Germany) for taking the SEM pictures and Stefan Schreiber (our institute) for implementing PIA.

Financial support. This research was funded by the Deutsche Forschungsgemeinschaft (DFG) (grant no. GE 779/38-1).

This open-access publication was funded by the Technische Universität Dresden (TUD).

Review statement. This paper was edited by Winfried Vonau and reviewed by two anonymous referees.

\section{References}

Dušek, K. and Patterson, D.: Transition in swollen polymer networks induced by intramolecular condensation, J. Polym Sci. Pol. Phys., 6, 1209-1216, 1968.

Erfkamp, J., Guenther, M., and Gerlach, G.: Hydrogel-based piezoresistive sensor for the detection of ethanol, J. Sens. Sens. Syst., 7, 219-226, https://doi.org/10.5194/jsss-7-219-2018, 2018.

Franke, D. and Gerlach, G.: Swelling studies of porous and nonporous semi-IPN hydrogels for sensor and actuator applications, Micromachines, 11, 425, https://doi.org/10.3390/mi11040425, 2020.

Franke, D., Binder, S., and Gerlach, G.: Performance of fastresponsive, porous crosslinked poly ( $N$-isopropylacrylamide) in a piezoresistive microsensor, IEEE Sens. Lett., 1, 1500904, https://doi.org/10.1109/LSENS.2017.2773626, 2017.

Guenther, M. and Gerlach, G.: Hydrogels for chemical sensors, in: Hydrogel sensors and actuators, eds.: Gerlach, G. and Arndt, K.F., Springer, Berlin, Heidelberg, Germany, 165-195, 2009.

Guenther, M., Gerlach, G., Corten, C., Kuckling, D., Müller, M., Shi, Z., Sorber, J., and Arndt, K.-F.: Application of polyelectrolytic temperature-responsive hydrogels in chemical sensors, Macromol. Symp., 254, 314-321, https://doi.org/10.1002/masy.200750846, 2007.
Hentze, H.-P. and Antonietti, M.: Template synthesis of porous organic polymers, Curr. Opin. Solid State Mater. Sci. 5, 343-353, https://doi.org/10.1016/S1359-0286(01)00008-0, 2001.

ImageJ software: https://imagej.nih.gov/ij/ (last access: 19 June 2020), 2018.

Kuckling, D., Arndt, K.-F., and Richter, A.: Temperature and $\mathrm{pH}$ dependent swelling behavior of poly( $N$-isopropylacrylamide)copolymer and their use in flow control, Macromol. Mater. Eng., 288, 144-151, https://doi.org/10.1002/mame.200390007, 2003.

Nayak, S. and Lyon, L. A.: Soft nanotechnology with soft nanoparticles (esp. Figs. 2 and 6 and associated text passages), Angew. Chem. Int. Edit., 44, 7686-7708, https://doi.org/10.1002/anie.200501321, 2005.

Orakdogen, N. and Okay, O.: Reentrant conformation transition in poly $(N, N$-dimethylacrylamide) hydrogels in water-organic solvent mixtures, Polymer, 47, 561-568, https://doi.org/10.1016/j.polymer.2005.11.066, 2006.

Pavel, F. M.: Microemulsion polymerization, J. Disper. Sci. Technol., 25, 1-16, https://doi.org/10.1081/DIS-120027662, 2004.

Richter, A.: Hydrogels for actuators, in: Hydrogel sensors and actuators, eds.: Gerlach, G. and Arndt, K.-F., Springer, Berlin, Heidelberg, Germany, 221-248, 2009.

Richter, A. and Paschew, G.: Optoelectrothermic control of highly integrated polymer-based MEMS applied in an artificial skin, Adv. Mater., 21, 979-983, https://doi.org/10.1002/adma.200802737, 2009.

Richter, A., Klatt, S., Paschew, G., and Klenke, C.: Micropumps operated by swelling and shrinking of temperature-sensitive hydrogels, Lab Chip, 9, 613-618, https://doi.org/10.1039/b810256b, 2009.

Saito, S., Konno, M., and Inomata, H.: Volume phase transition of $N$-alkylacrylamide gels, in: Responsive gels: Volume transitions I, ed.: Dušek, K., Springer, Berlin, Germany, 207-232, 1993.

Schulz, V., Zschoche, S., Zhang, H. P., Voit, B., and Gerlach, G.: Macroporous smart hydrogels for fast-responsive piezoresistive chemical microsensors, Procedia Engineer., 25, 1141-1144, https://doi.org/10.1016/j.proeng.2011.12.281, 2011.

Sjöblom, J., Lindberg, R., and Friberg, S. E. : Microemulsions - phase equilibria characterization, structures, applications and chemical reactions, Adv. Colloid Interfac., 65, 125-287, 1996.

Tanaka, T.: Collapse of gels and the critical endpoint, Phys. Rev. Lett., 40, 820-823, 1978.

Tiddy, G. J. T.: Surfactant-water liquid crystal phases, Phys. Rep., 57, 1-46, 1980. 